\title{
Magnetic monopole search with the MACRO detector at Gran Sasso
}

\section{MACRO Collaboration}

M. Ambrosio ${ }^{\ell}$, R. Antolini ${ }^{\mathrm{g}}$, G. Auriemma ${ }^{\mathrm{n}, 1}$, R. Baker ${ }^{\mathrm{k}}$, A. Baldini ${ }^{\mathrm{m}}$, G.C. Barbarino ${ }^{\ell}$, B.C. Barish ${ }^{d}$, G. Battistoni ${ }^{f, 2}$, R. Bellotti ${ }^{a}$, C. Bemporad ${ }^{m}$, P. Bernardini ${ }^{j}$, H. Bilokon ${ }^{f}$,

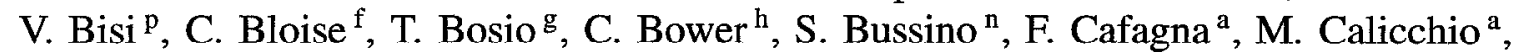
D. Campana ${ }^{\ell}$, M. Carbonif ${ }^{f}$, M. Castellano ${ }^{a}$, S. Cecchini ${ }^{\text {b, }}$, , F. Cei ${ }^{\mathrm{m}, 4}$, V. Chiarella ${ }^{\mathrm{f}}$, A. Corona ${ }^{n}$, S. Coutu ${ }^{k}$, G. De Cataldo ${ }^{a}$, H. Dekhissi ${ }^{\text {b, }}$, C. De Marzo ${ }^{a}$, I. De Mitri ${ }^{i}$, M. De Vincenzi ${ }^{n, 6}$, A. Di Credico ${ }^{g}$, O. Erriquez ${ }^{a}$, R. Fantini ${ }^{\text {b }}$, C. Favuzzi ${ }^{\text {a }}$, C. Forti ${ }^{\text {, }}$, P. Fusco $^{a}$, G. Giacomelli ${ }^{\text {b }}$, G. Giannini ${ }^{\mathrm{m}, 7}$, N. Giglietto ${ }^{a}$, M. Goretti ${ }^{\mathrm{d}, \mathrm{n}}$, M. Grassi ${ }^{\mathrm{m}}$, L. Gray ${ }^{g}$, P. Green ${ }^{11}$, A. Grillog, F. Guarino ${ }^{\ell}$, P. Guarnaccia ${ }^{a}$, C. Gustavino ${ }^{g}$, A. Habig ${ }^{h}$, K. Hanson ${ }^{\mathrm{k}}$, A. Hawthorne ${ }^{\mathrm{h}}$, R. Heinz ${ }^{\mathrm{h}}$, J.T. Hong ${ }^{\mathrm{c}}$, E. Iarocci ${ }^{\mathrm{f}, 8}$, E. Katsavounidis ${ }^{d}$, E. Kearns ${ }^{\text {c }}$, S. Kyriazopoulou ${ }^{\text {d }}$, E. Lamanna ${ }^{n}$, C. Lane ${ }^{\text {e }}$, D.S. Levin ${ }^{k}$, P. Lipari ${ }^{n}$, G. Liu $^{d}$, R. Liu ${ }^{d}$, N.P. Longley ${ }^{d, 12}$, M.J. Longo ${ }^{k}$, G. Ludlam ${ }^{c}$, G. Mancarella ${ }^{j}$, G. Mandrioli ${ }^{b}$, A. Margiotta Neri ${ }^{b}$, A. Marini ${ }^{f}$, D. Martello ${ }^{j}$, A. Marzari-Chiesa ${ }^{p}$, M.N. Mazziotta ${ }^{a}$, D.G. Michael ${ }^{\text {d}}$, S. Mikheyev ${ }^{\text {g, }}$, L. Miller ${ }^{\text {h }}$, P. Monacelli ${ }^{i}$, T. Montaruli ${ }^{a}$, M. Monteno ${ }^{\text {, }}$,

S. Mufson ${ }^{\text {h }}$ J. Musser ${ }^{\mathrm{h}}$, D. Nicoló ${ }^{\mathrm{m}, 4}$, R. Nolty ${ }^{\mathrm{d}}, \mathrm{C}$. Okada ${ }^{\mathrm{c}}$, C. Orth ${ }^{\mathrm{c}}$, G. Osteria ${ }^{\ell}$,

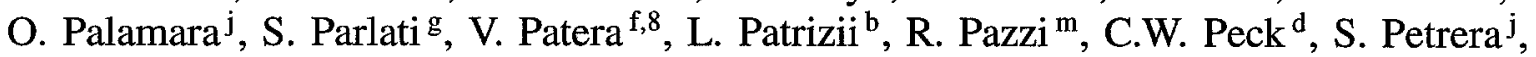
N.D. Pignatano ${ }^{d}$, P. Pistilli ${ }^{j}$, V. Popa ${ }^{b, 10}$, A. Rainó ${ }^{a}$, J. Reynoldson ${ }^{g}$, F. Ronga ${ }^{f}$, U. Rubizzo ${ }^{\ell}$, A. Sanzgiri ${ }^{\circ}$, F. Sartogo ${ }^{n}$, C. Satriano ${ }^{\text {n, }}$, L. Satta ${ }^{\text {f, }, ~}$, E. Scapparone ${ }^{g}$, K. Scholberg ${ }^{\mathrm{d}}$, A. Sciubba ${ }^{\mathrm{f}, 8}$, P. Serra-Lugaresi ${ }^{\mathrm{b}}$, M. Sioli ${ }^{\mathrm{g}}$, M. Sitta ${ }^{\mathrm{p}}$, P.F. Spada ${ }^{\mathrm{b}}$, P. Spinelli ${ }^{a}$, M. Spinetti ${ }^{\mathrm{f}}$, M. Spurio ${ }^{\mathrm{b}}$, R. Steinberg ${ }^{\mathrm{e}}$, J.L. Stone ${ }^{\mathrm{c}}$, L.R. Sulak ${ }^{\mathrm{c}}$, A. Surdo ${ }^{\mathrm{j}}$,

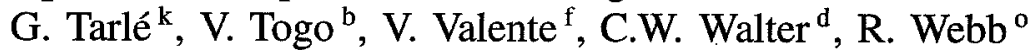

a Dipartimento di Fisica dell'Università di Bari and INFN, 70126 Bari, Italy

' Dipartimento di Fisica dell'Università di Bologna and INFN, 40126 Bolognu, Iialy

c Physics Department, Boston University, Boston, MA 02215, USA

d California Institute of Technology, Pasadena, CA 91125, USA

e Department of Physics, Drexel University, Philadelphia, PA 19104, USA

f Laboratori Nazionali di Frascati dell'INFN, 00044 Frascati (Roma), Italy

g Laboratori Nazionali del Gran Sasso dell'INFN, 67010 Assergi (L'Aquila), Italy

${ }^{\mathrm{h}}$ Depts. of Physics and of Astronomy, Indiana University, Bloomington, IN 47405, USA

i Dipartimento di Fisica dell'Università dell'Aquila and INFN, 67100 L'Aquila, Italy

j Dipartimento di Fisica dell'Università di Lecce and INFN, 73100 Lecce, Italy

${ }^{k}$ Department of Physics, University of Michigan, Ann Arbor, MI 48109, USA

${ }^{\ell}$ Dipartimento di Fisica dell'Università di Napoli and INFN, 80125 Napoli, Italy 


\author{
m Dipartimento di Fisica dell'Università di Pisa and INFN, 56010 Pisa, Italy \\ "Dipartimento di Fisica dell'Università di Roma "La Sapienza" and INFN, 00185 Roma, Italy \\ - Physics Department, Texas A\&M University, College Station, TX 77843, USA \\ ${ }^{\mathrm{P}}$ Dipartimento di Fisica Sperimentale dell'Università di Torino and INFN, 10125 Torino, Italy
}

Received 12 May 1997

Editor: K. Winter

\begin{abstract}
In this letter we present the results of the search for massive magnetic monopoles in the penetrating cosmic ray radiation using the various subdetectors of the MACRO apparatus, during the period 1989-1995. Flux limits are given for the $\beta=v / c$ range $4 \times 10^{-5}<\beta<1$; for $10^{-4}<\beta<10^{-1}$ the limits are below the Parker bound, $\sim 10^{-15} \mathrm{~cm}^{-2} \mathrm{~s}^{-1} \mathrm{sr}^{-1}$, (C) 1997 Published by Elsevier Science B.V.
\end{abstract}

\section{Introduction}

Grand Unified Theories (GUTs) of the electroweak and strong interactions predict the existence of massive $\left(>10^{16} \mathrm{GeV}\right.$ ) magnetic monopoles [1]. It is expected that such magnetic monopoles might have been produced immediately after the Big Bang. Since that time, the magnetic monopoles would have cooled down to very low velocities. Subsequent gravitational or magnetic mechanisms could accelerate them to higher velocities. Monopoles trapped around the Earth are expected to travel with $\beta=v / c \simeq$ few times $10^{-5}$, while monopoles trapped around the Sun and the Galaxy have betas $\sim 10^{-4}$ and $\sim 10^{-3}$, respectively. These "virial" velocities are slightly smaller than the escape velocities. Monopoles with smaller masses and larger velocities are conceivable [2].

\footnotetext{
${ }^{1}$ Also Università della Basilicata, 85100 Potenza, Italy.

2 Also INFN Milano, 20133 Milano, Italy.

${ }^{3}$ Also Istituto TESRE/CNR, 40129 Bologna, Italy.

${ }^{4}$ Also Scuola Normale Superiore di Pisa, 56010 Pisa, Italy.

${ }^{5}$ Also Faculty of Sciences, University Mohamed I, B.P. 424 Oujda, Morocco.

${ }^{6}$ Also Dipartimento di Fisica, Università di Roma Tre, Roma, Italy.

${ }^{7}$ Also Università di Trieste and INFN, 34100 Trieste, Italy.

${ }^{8}$ Also Dipartimento di Energetica, Università di Roma, 00185 Roma, Italy.

${ }^{9}$ Also Institute for Nuclear Research, Russian Academy of Science, 117312 Moscow, Russia.

${ }^{10}$ Also Institute for Gravity and Space Sciences, 76900 Bucharest, Romania.

${ }^{11}$ Sandia National Laboratory, Albuquerque, NM 87185, USA.

${ }^{12}$ Swarthmore College, Swarthmore, PA 19081, USA.
}

One of the primary aims of the MACRO detector, located in the underground Hall B of Laboratori Nazionali del Gran Sasso in Italy, is the search for such magnetic monopoles at a level well below the Parker bound (about $10^{-15} \mathrm{~cm}^{-2} \mathrm{~s}^{-1} \mathrm{sr}^{-1}$, calculated on the basis of the survival of the galactic magnetic field) [3] and for a large range of velocities, $4 \times 10^{-5}<\beta<1$.

The MACRO detector has global dimensions of $76.6 \times 12 \times 9.3 \mathrm{~m}^{3}$ and is divided longitudinally in six similar supermodules and vertically in a lower $(4.8 \mathrm{~m}$ high) and an upper part ( $4.5 \mathrm{~m}$ high). For the full description of the detector see Ref. [4]. Unlike previous monopole searches, the MACRO experiment employs simoultaneously three different subdetectors: planes of liquid scintillation counters, planes of limited streamer tubes, each with electronics designed to identify both fast and slow penetrating particles, and planes of passive nuclear track detectors. A single event may have distinctive and multiple signatures in the three subdetectors. This redundancy is important for rare events.

It should be pointed out that special care was taken to ensure that the three types of subdetectors were sensitive to low $\beta$ particles. A measurement of the light yield in plastic scintillators of protons moving with velocities $10^{-4}<\beta<10^{-2}$ allowed inference of the light yield of magnetic monopoles with similar velocities. This helped to assess the performance and the sensitivity of searches for monopoles using scintillators in the low velocity regime [5]. The use of a helium-n-pentane gas mixture $(73 \% \mathrm{He}$ and $27 \% \mathrm{n}$ pentane) in the streamer tubes allows detection, with essentially $100 \%$ efficiency, of slow monopoles exploiting the Drell and Penning effects $[4,6,15]$. 
The CR39 nuclear track subdetector was calibrated with slow and fast ions, checking that the response was dependent only on the restricted energy loss [7].

In the present letter a direct search for magnetic monopoles is reported. The limits are obtained under the following hypotheses: a) bare monopoles with unit Dirac magnetic charge; b) cross section for monopole catalysis of nucleon decay less than few millibarn; c) isotropic flux. The last hypothesis is valid for magnetic monopoles with mass $\geq 10^{17} \mathrm{GeV}$ and for any $\beta>$ $10^{-4}$. For monopoles with lower masses, which may not traverse the earth, the flux limits may be up to a factor of two larger. In the future more extended analyses will be reported.

The data were collected in the period 1989-1995, during which the implementation of the detector was underway. During this period the single subdetectors were not necessarily all active at the same time.

The use of different triggers allowed to cover the whole $\beta$-region from $\beta \simeq 4 \times 10^{-5}$ to $\beta \simeq 1$. No monopole candidate was observed. We refer to Refs. [4-7,9-12] for details on the subdetectors, of their performances in the searches for magnetic monopoles and on the specific analysis techniques. In this letter we summarize the limits derived from the analyses of all the data collected prior to August 1995. Some of these limits have already been published in refereed journals $[5,6,10]$

\section{Searches using the liquid scintillator subdetector}

The liquid scintillation subdetector consists of three horizontal planes of scintillators and of vertical walls covering the sides and the lower ends of the detector. In the present analyses only the lower part of the detector has been used.

In the liquid scintillator subdetector we have employed different specialized triggers covering specific velocity regions. The corresponding searches have been grouped into searches for low velocity $\left(1.8 \times 10^{-4}<\beta<6 \times 10^{-3}\right)$, medium velocity $\left(1.2 \times 10^{-3}<\beta<10^{-1}\right)$ and high velocity $\left(10^{-1}<\beta<1\right)$ monopoles.

\section{Searches for low velocity monopoles}

A search for slowly moving magnetic monopoles in the penetrating cosmic radiation was based on a cus- tom made trigger circuit (the SMT) and 20 and 50 $\mathrm{MHz}$ Wave Form Digitizers (WFD). A first data set was collected from October 1989 to November 1991, for a live-time of $\mathbf{5 4 2}$ days using the first supermodule of MACRO. This search was published in Ref. [5]. The analysis required the SMT to be present in more than one scintillator layer while the fast particle trigger was used as an off-line veto. Visual scanning of the remaining events was performed. The analysis was sensitive to monopoles in the velocity range $1.8 \times 10^{-4}<\beta<4 \times 10^{-3}$. From the absence of candidates we established an upper limit on the magnetic monopole flux at the $90 \%$ confidence level (C.L.) of $5.6 \times 10^{-15} \mathrm{~cm}^{-2} \mathrm{~s}^{-1} \mathrm{sr}^{-1}$ in the region $1.8 \times 10^{-4}<$ $\beta<3 \times 10^{-3}$. The upper velocity limit was determined by the minimum pulse train duration required by the trigger circuit (see Table 1, column 2, and Fig. 1 , curve "Scintill. A").

In the period December 1992 through June 1993, in 163.5 days of live-time, were collected $2.6 \times 10^{6}$ slow monopole triggers using all the lower six supermodules. This search was based on the SMT to be present in more than one scintillator layer and a 40 $\mathrm{MHz}$ waveform digitizer system. The fast particle trigger was used as an off-line veto. About 8000 candidate events were selected. A "wavelet transform" method was used for particle identification [12]. Using various features of the Haar [13] decomposition of the waveforms, simple conditions were derived by means of which more than $80 \%$ of the coincidence events were rejected. The efficiency of the algorithm was measured using LED-generated monopole-like events and Monte Carlo techniques. The remaining events were visually scanned and classified. No waveform was consistent with a slow magnetic monopole signature. On the basis of the above information, an upper flux limit at the $90 \%$ C.L. was established for $1.8 \times 10^{-4}<\beta<4 \times 10^{-3}$. The limit is $4.1 \times 10^{-15}$ $\mathrm{cm}^{-2} \mathrm{~s}^{-1} \mathrm{sr}^{-1}$ for $1.8 \times 10^{-4}<\beta<2.7 \times 10^{-3}$ (the limit is given in Table 1 , column 5 , and Fig. 1 , curve "Scintill. D").

Another analysis has been performed using the same data sample described above with a different waveform analysis method [12]. The monopole upper flux limit is included in the previous one for $\beta<3 \times 10^{-3}$; for the $3 \times 10^{-3}<\beta<6 \times 10^{-3}$ region, the limit is shown in Table 1, column 5, and in Fig. 1, "Scintill. D". 
Table 1

The $90 \%$ C.L. MACRO flux limits (in units of $10^{-15} \mathrm{~cm}^{-2} \mathrm{~s}^{-1} \mathrm{sr}^{-1}$ ) as a function of $\beta$ for an isotropic flux of $g=g D$ unagnetic monopoles assuming a catalysis cross section smaller than $10 \mathrm{mb}$. The limits discussed in Sections $2-4$ are given in columns two to seven and shown in Fig. 1. The single lowest limit is in column eight; the global MACRO limit discussed in Section 5 is given in the last column and shown in Fig. 2

\begin{tabular}{|c|c|c|c|c|c|c|c|c|}
\hline \multirow[b]{2}{*}{ Typical $\beta$} & \multicolumn{8}{|c|}{ Flux Limits $\left(10^{-15} \mathrm{~cm}^{-2} \mathrm{~s}^{-1} \mathrm{sr}^{-1}\right)$} \\
\hline & $\begin{array}{l}\text { Scintill. A } \\
\text { [5] }\end{array}$ & $\begin{array}{c}\text { Scintill. B } \\
\text { [11] }\end{array}$ & $\begin{array}{c}\text { Scintill. C } \\
{[10]}\end{array}$ & $\begin{array}{l}\text { Scintill. D } \\
\text { [12] }\end{array}$ & $\begin{array}{l}\text { Streamer } \\
\text { tubes [11] }\end{array}$ & $\begin{array}{c}\text { CR39 } \\
{[11]}\end{array}$ & $\begin{array}{l}\text { Single } \\
\text { lowest }\end{array}$ & Global \\
\hline $4.010^{-5}$ & & & & & & 6.8 & 6.8 & 6.8 \\
\hline $5.010^{-5}$ & & & & & & 5.3 & 5.3 & 5.3 \\
\hline $8.010^{-5}$ & & & & & & 4.3 & 4.3 & 4.3 \\
\hline $1.110^{-4}$ & & & & & & 4.2 & 4.2 & 4.2 \\
\hline $1.110^{-4}$ & & & & & 0.87 & 4.2 & 0.87 & 0.78 \\
\hline $1.410^{-4}$ & & & & & 0.87 & 4.7 & 0.87 & 0.79 \\
\hline $1.810^{-4}$ & & & & & 0.87 & 6.9 & 0.87 & 0.81 \\
\hline $1.810^{-4}$ & 5.6 & & & 4.1 & 0.87 & 6.9 & 0.87 & 0.68 \\
\hline $2.010^{-4}$ & 5.6 & & & 4.1 & 0.87 & 10. & 0.87 & 0.69 \\
\hline $2.510^{-4}$ & 5.6 & & & 4.1 & 0.87 & 100 & 0.87 & 0.70 \\
\hline $1.210^{-3}$ & 5.6 & & & 4.1 & 0.87 & & 0.87 & 0.70 \\
\hline $1.210^{-3}$ & 5.6 & 1.0 & & 4.1 & 0.87 & & 0.87 & 0.61 \\
\hline $2.110^{-3}$ & 5.6 & 1.0 & & 4.1 & 0.87 & 100 & 0.87 & 0.61 \\
\hline $2.710^{-3}$ & 5.6 & 1.0 & & 4.1 & 0.87 & 12.6 & 0.87 & 0.61 \\
\hline $3.010^{-3}$ & 5.6 & 1.0 & & 4.8 & 0.87 & 10. & 0.87 & 0.62 \\
\hline $4.010^{-3}$ & 12.7 & 1.0 & & 6.4 & 0.87 & 6.8 & 0.87 & 0.66 \\
\hline $5.010^{-3}$ & & 1.0 & & 8.0 & 0.87 & 5.4 & 0.87 & 0.66 \\
\hline $5.010^{-3}$ & & 1.0 & . & 8.0 & 0.87 & 5.4 & 0.87 & 0.93 \\
\hline $6.010^{-3}$ & & 1.0 & & 14.6 & & 4.9 & 1.0 & 0.92 \\
\hline $1.010^{-2}$ & & 1.0 & & & & 3.7 & 1.0 & 0.89 \\
\hline $2.010^{-2}$ & & 1.0 & & & & 3.2 & 1.0 & 0.86 \\
\hline $5.010^{-2}$ & & 1.0 & & & & 2.8 & 1.0 & 0.84 \\
\hline 0.1 & & 1.0 & & & & 2.8 & 1.0 & 0.84 \\
\hline 0.1 & & & 4.4 & & & 2.8 & 2.8 & 1.8 \\
\hline 1 & & & 4.4 & & & 2.7 & 2.7 & 1.8 \\
\hline
\end{tabular}

\section{Searches for medium velocity monopoles}

A monopole search was performed in this range using the electronics designed primarily for the detection of supernova neutrinos $[4,14]$. It uses the Pulse Height Recorder and Synchronous Encoder (PHRASE) system, which includes a $100 \mathrm{MHz}$ WFD system. Although the waveforms saturate at a pulse height equivalent to about $15 \mathrm{MeV}$, the shape of the unsaturated portion can be used to reconstruct the energy of the saturated pulse. The data sample used in Refs. $[9,10]$ was collected between Octuber 1989 and June 1993 in various configurations of the apparatus. The event selection required two hits in two different scintillator faces that represented an energy release of at least 10 $\mathrm{MeV}$ in each face. A minimum separation of $2 \mathrm{~m}$ was required for the hits in the two faces while the soft- ware cut of $10^{-1} \mathrm{c}$ in velocity was imposed in order to reject the tail of the cosmic ray muon velocity distribution. The apparent velocity between the two planes is reconstructed using the scintillator time information. The surviving events were divided in two sub-ranges: $1.2 \times 10^{-3}<\beta<5 \times 10^{-3}$ and $5 \times 10^{-3}<\beta<$ $10^{-1}$. Candidates in the first sample were rejected by comparing the duration of the scintillation light pulse (measured by the PHRASE WFD) with the expected one (computed using the apparent velocity); candidates belonging to the second sample were rejected on the basis of the measured energy loss compared with the one expected for monopoles. Candidates with velocities around $\beta \sim 5 \times 10^{-3}$ were examined using both techniques. No event survived these criteria. With the help of the tracking system a large fraction $(\sim 80 \%)$ of the candidates was found to arise from 


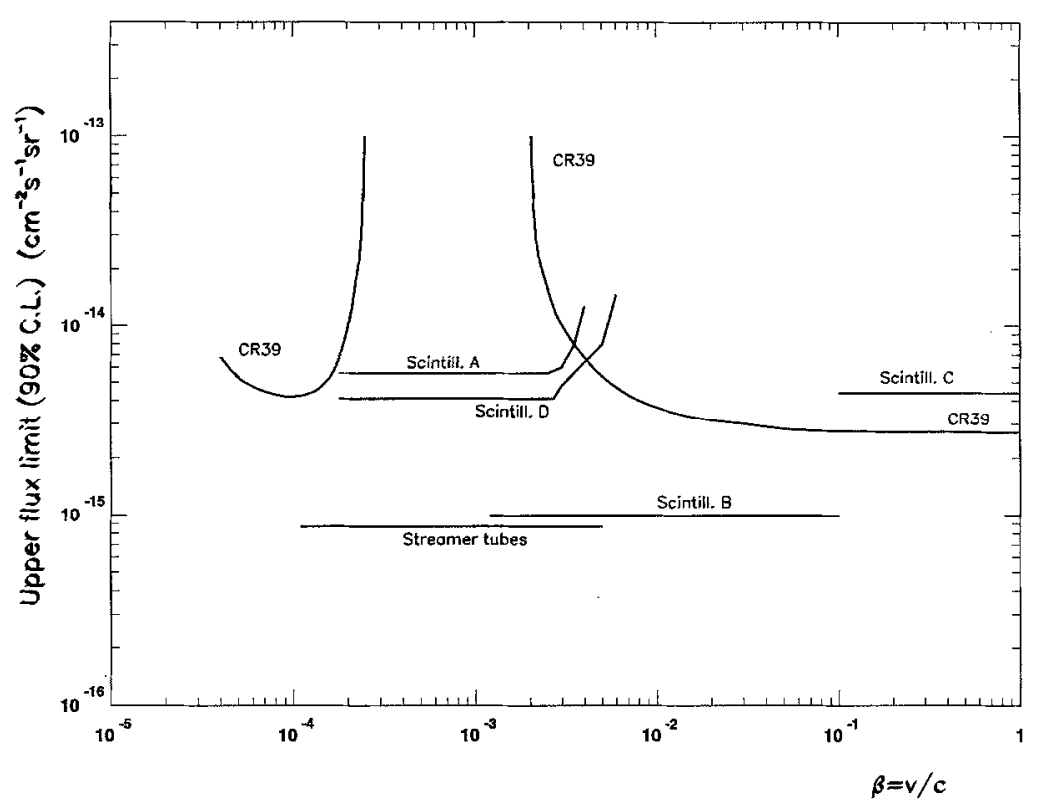

Fig. 1. The $90 \%$ C.L. upper limits for an isotropic flux of bare $g=g_{D}$ magnetic monopoles, assuming a catalysis cross section smaller than $10 \mathrm{mb}$, obtained using the various MACRO subdetectors and different electronics, as described in the text.

cosmic ray muons stopping in the detector and decaying to an electron. The remaining fraction was consistent with accidental coincidences between cosmic rays and natural radioactivity events. From the absence of magnetic monopole candidates, a $90 \%$ C.L. upper flux limit of $4 \times 10^{-15} \mathrm{~cm}^{-2} \mathrm{~s}^{-1} \mathrm{sr}^{-1}$ was established $[9,10]$. The analyses has been extended to data taken until the end of July 1995 [11]; the limit is now $10^{-15}$ $\mathrm{cm}^{-2} \mathrm{~s}^{-1} \mathrm{sr}^{-1}$ (see Table 1, column 3, and Fig. 1, curve "Scintill. B").

\section{Searches for high velocity monopoles}

This search was mainly based on the liquid scintillator subdetector using the trigger and the energy reconstruction given by the ERP system $[4,10,11]$. Particular care was taken to obtain a good absolute energy loss calibration. The search used data taken from December 1992 to July 1993, during which the entire lower MACRO detector was operational. The energy loss was measured in two scintillator layers. The requirement of a high energy deposition in both counters $(E>600 \mathrm{MeV}$ ) rejected most of the muon events. Then the WFD and streamer tube tracking information were used to reject non-monopole events, requiring a single clean track and a waveform compatible with a monopole signal. With no candidate event found, an upper flux limit for fast moving magnetic monopoles at the $90 \%$ C.L. of $4.4 \times 10^{-15} \mathrm{~cm}^{-2} \mathrm{~s}^{-1} \mathrm{sr}^{-1}$ for $10^{-1}<\beta<1$ was established (see Table 1, column 4 , and Fig. 1, curve "Scintill. C").

\section{Search using the streamer tube subdetector}

The streamer tube subdetector of the lower MACRO consists of 10 horizontal planes and 6 vertical planes covering the vertical sides as a closed box. In this analysis only the horizontal streamer planes of the lower MACRO have been used for triggering, while for measuring the particle velocity all the hit planes have been used.

The published analysis was based on the search for single tracks in both wire and strip views and on the velocity measurements of the candidates [6]. The analysed data sample was collected from February 1992 to January 1993. The trigger and analysis were verified to be velocity independent; the overall efficiency was estimated by computing the ratio of the rate of single 


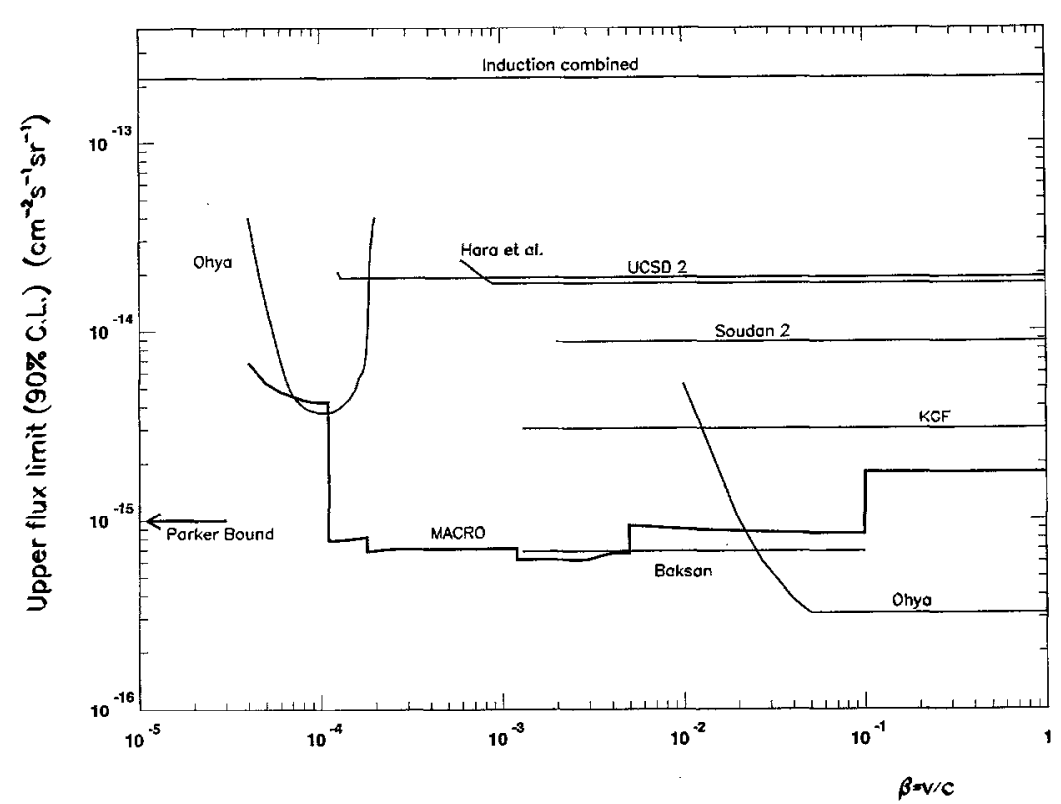

Fig. 2. The $90 \%$ C.L. upper limits for an isotropic flux of bare $g=g_{D}$ magnetic monopoles assuming a catalysis cross section smaller than $10 \mathrm{mb}$, obtained by MACRO (global limit) and by other experiments: Induction combined [16], UCSD2 [17], Soudan 2 [18], Baksan [19], Ohya [20], KGF [21], Hara et al. [22].

muons reconstructed by this analysis to that expected; its typical value was over $90 \%$. A Monte Carlo simulation including all the geometrical and trigger requirements was used to compute the detector acceptance. No candidate remained. The limit has been updated with all the data taken until July 31,1995 . The upper flux limit is now $\Phi_{M}<8.7 \times 10^{-16} \mathrm{~cm}^{-2} \mathrm{~s}^{-1} \mathrm{sr}^{-1}$ at $90 \%$ C.L. for $1.1 \times 10^{-4}<\beta<5 \times 10^{-3}[6,11]$. (see Table 1, column 6, and Fig. 1, curve "Streamer tubes") [11].

\section{Search using the nuclear track subdetector}

The nuclear track subdetector is located horizontally in the middle of the lower MACRO structure, and vertically on the east wall and on the lower part of the north wall. It covers a surface of $1263 \mathrm{~m}^{2}$ and the total acceptance for fast magnetic monopoles is about $7100 \mathrm{~m}^{2} \mathrm{sr}$. The subdetector is used as a stand alone subdetector and in a "triggered mode" by the scintillator and streamer tube systems. In the last three years, about $24 \mathrm{~m}^{2} /$ year of track etch detector were extracted. The analysis was limited to the CR39 de- tector only. The method of searching for magnetic monopoles and the determination of the geometrical and detection efficiencies are reported in detail in [7]. A total surface area of $75.8 \mathrm{~m}^{2}$ of CR39 has been analyzed, with an average exposure time of 5.6 years. No candidate was found; the $90 \%$ C.L. upper limits on the magnetic monopole flux is at the level of $2.7 \times 10^{-15} \mathrm{~cm}^{-2} \mathrm{~s}^{-1} \mathrm{sr}^{-1}$ at $\beta \sim 1$, and $4.2 \times 10^{-15}$ $\mathrm{cm}^{-2} \mathrm{~s}^{-1} \mathrm{sr}^{-1}$ at $\beta \sim 10^{-4}$, (see Table 1, column 7, and Fig. 1, curves "CR39") $[9,11]$.

\section{Discussion and conclusions}

No candidates were found in any of the above mentioned searches. The corresponding 90\% C.L. flux limits are given in Table 1 and shown in Fig. 1 as function of $\beta$. The single lowest limit in each $\beta$-interval is indicated in the eighth column of Table 1 . Since any one of the subdetectors may rule out, within its own acceptance and sensitivity, a potential candidate, a global limit has been derived by combining together the limits obtained by the single subdetectors. The combination has been done taking into account the overlap in time 
and in space. The full beta interval has been divided in beta slices sufficient to characterize the change in individual acceptance. In each beta slice, also the total MACRO running time has been divided in intervals $T_{i}$ of live time sufficient to characterize the change in acceptance due to different subdetector configurations. Then the time integrated acceptance $A_{i}$ has been calculated multiplying the live time $T_{i}$ by the acceptance of the subdetectors running in the period $T_{i}$. In each beta slice the $A_{\text {total }}$ factor is calculated simply summing the $A_{i}$. The global $90 \%$ c.1. MACRO upper limit is then $\Phi=2.3 / A_{\text {total }}$.

The present global MACRO limit is given, as a function of $\beta$, in the last column of Table 1 and in Fig. 2. In Fig. 2 it is compared with the results obtained by other experiments which searched for bare magnetic monopoles with $g=g_{D}$ and $\sigma_{\text {cat }}<$ few mb $[16,22]$. The indirect mica limits $[23,24]$ are not included in the present discussion as they apply to magnetic monopole-nucleus bound states.

In the range $10^{-4}<\beta<10^{-1}$ the MACRO monopole limit is at the level of $0.61-0.9 \times$ $10^{-15} \mathrm{~cm}^{-2} \mathrm{~s}^{-1} \mathrm{sr}^{-1}$. In $10^{-4}<\beta<10^{-3}$ the limit is for the first time below the Parker bound [3]. In this work the data have been collected with separate subsystems of the full apparatus and combined to produce the best overall limit. In the future we will improve on this limit while exploiting the redundancy features of the combined subsystems.

\section{Acknowledgements}

We gratefully acknowledge the support of the director and of the staff of the Laboratori Nazionali del Gran Sasso and the invaluable assistance of the technical staff of the Institutions participating in the experiment. We thank the Istituto Nazionale di Fisica Nucleare (INFN), the US Department of Energy and the US National Science Foundation for their generous support of the MACRO experiment. We thank INFN for providing fellowships and grants (FAI) for non Italian citizens.

\section{References}

[1] G. 't Hooft, Nucl. Phys. B 79 (1974) 276; A.M. Polyakov, IETP Lett. 20 (1974) 194; J. Preskill, Phys. Rev. Lett. 43 (1979) 1365.
[2] A. De Rujula, Nucl. Phys. B 435 (1995) 257.

[3] M.S. Turner, E.M. Parker and T.J. Bogdan, Phys. Rev. D 26 (1982) 1926;

An improved bound is given, in: F.C. Adams et al., Phys. Rev. Lett. 70 (1993) 2511.

[4] MACRO Collaboration, S. Ahlen et al., Nucl. Instr. \& Meth. A 324 (1993) 337.

[5] MACRO Collaboration, S. Ahlen et al., Phys. Rev. Lett. 72 (1994) 608.

[6] MACRO Collaboration, S. Ahlen et al., Astrop. Phys. 4 (1995) 33.

[7] MACRO Collaboration, S. Ahlen et al., Search for magnetic monopoles with the MACRO track etch detector, LNGS94/115 (1994).

[8] G.G. Callan, Phys. Rev. D 26 (1982) 2058; V.A. Rubakov, JETP Lett. 33 (1981) 644; Nucl. Phys. B 203 (1982) 311.

[9] MACRO Collaboration, M. Ambrosio et al., Search for fast magnetic monopoles with the MACRO scintillator system, LNGS-95/45, and Search for GUT monopoles with the MACRO streamer tube and track etch systerns, LNGS-95/48; papers presented at the $24^{\text {th }}$ ICRC, Rome (1995).

[10] MACRO Collaboration, M. Ambrosio et al., (The performance of the MACRO liquid scintillator in the search for magnetic monopoles with $10^{-3}<\beta<1$ ), Astrop. Phys. 6 (1997) 113.

[11] MACRO Collaboration, M. Ambrosio et al. (MACRO limits for $g=g_{D}$ magnetic monopoles) MACRO/PUB $96 / 2$ (1996);

Paper presented by F. Guarino at the 28th Int. Conf. High En. Phys., Warsaw (1996).

[12] MACRO Collaboration, M. Ambrosio et al., (Search for slow GUT magnetic monopoles with the MACRO scintillator detector), MACRO/PUB 96/1 (1996);

Paper presented by E. Katsavounidis at the DPF96 Meeting of the Division of Particles and Fields of the American Physical Society, Minneapolis, Minn (1996).

[13] A. Haar, Math. Ann. 69 (1910) 331.

[14] MACRO Collaboration, M. Ambrosio et al., Astrop. Phys. 1 (1992) 11.

[15] S. Drell et al., Phys. Rev. Lett. 50 (1983) 644.

[16] S. Bermon et al., Phys. Rev. Lett. 64 (1990) 839.

[17] K.N. Buckland et al., Phys. Rev. D 41 (1990) 2726.

[18] J.L. Thron et al., Phys. Rev. D 46 (1992) 4846.

[19] E.N. Alexeyev et al. (A search for superheavy magnetic monopoles by the Baksan underground scintillation telescope), $21^{\text {st }}$ ICRC, Adelaide, Vol. 10 (1990) 83. New limits may be available soon.

[20] S. Orito et al., Phys. Rev. Lett. 66 (1991) 1951.

[21] H. Adarkar et al., (Kolar gold field monopole experiment), $21^{\text {st }}$ ICRC, Adelaide (1990) p. 95.

[22] T. Hara et al. (A search experiment for slow moving monopoles $\left(\beta \geqslant 2 \times 10^{-4}\right)$ using a helium proportional counters array), $21^{\text {st }}$ ICRC, Adelaide (1990) p. 79.

[23] P.B. Price and M.H. Salamon, Phys. Rev. Lett. 56 (1986) 1226.

[24] D. Ghosh and S. Chatterjea, Europhys. Lett. 12 (1990) 25. 